\title{
Anterior Thoracolumbar Fusion with Titanium Mesh Cage
}

\author{
Jae-Eun Choi, Jung-Chul Lim", Jae-Hyun Lee and Soo-Han Kim \\ Department of Neurosurgery, Chonnam National University Hospital and Medical School, \\ ${ }^{1}$ Department of Neurosurgery, Seonam University Nam-Gwang Hospital, Gwangiu, Korea
}

\begin{abstract}
Destructions of vertebral body due to various medical situations including acute fracture, tumor, post-traumatic deformity and infection lead to progressive destruction of vertebral body, kyphotic deformities and further neurological defecits. Titanium mesh cages (TMCs) with cancellous autograft bone after corpectomy of the thoracolumbar spine provide immediate structural support to the anterior column. The aim of this study was to evaluate the radiological, neurological and clinical outcomes of the patients with the TMCs-bone graft composite after thoracolumbar corpectomy. Sixteen patients underwent reconstruction using titanium mesh cages in thoracolumbar corpectomy between July 2000 and February 2005. The radiological and clinical course was documented over a mean follow-up duration of 28.2 months. The degree of kyphosis, construct height and the subsidence of the cage in relation to the vertebral endplates were measured preoperatively, early postoperatively, and at the latest follow-up. The mean kyphotic angle of $3.9^{\circ}$ before surgery was reduced to $-2.6^{\circ}$ immediately after surgery, and at the last follow-up to be $3.4^{\circ}$. There was a significant difference between the preoperative versus postoperative kyphotic angle $(p=0.003)$. The mean construct height of the involved vertebrae before surgery was $41.6 \mathrm{~mm}$ and the mean construct height immediate after surgery and at follow-up were 47.9 and $41.5 \mathrm{~mm}$, respectively. There was a significant difference between the preoperative versus postoperative construct height $(p<0.0001)$. But there was no significant difference between the preoperative versus follow-up in kyphotic angle and construct height. The mean subsidence was $5.7 \mathrm{~mm}$. However, there was no case of severe collapse or significant recurrence of deformity. In no patients, significant neurological deterioration was detected after surgery, and neither with major complications. This study demonstrated that TMCs after thoracolumbar corpectomy is a successful adjunct for anterior vertebral column reconstruction. In our cases, TMCs with anterior instrumentation allows a good structural support and maintain spinal alignment.
\end{abstract}

Keywords: Surgical mesh; Thoracic surgery; Lumbar vertebrae; Spinal fusion

\section{Introduction}

Destructions of vertebral body due to various medical situations including acute fracture, tumor, post-traumatic deformity and infection lead to progressive destruc-

Accepted for Publication: November 13, 2008

*Corresponding author: Jae-Eun Choi, 501-757, Department of Neurosurgery, Chonnam National University Hospital, Phone: 062-220-6606, FAX: 062224-9865, E-mail: stylish74@hanmail.net tion of vertebral body, kyphotic deformities and further neurological deficits. ${ }^{1-5}$ Anterior approach provides a more direct and complete decompression of the spinal canal, potentially allowing a better neurological outcome. ${ }^{6}$ So surgical corpectomy of the compromised anterior column requires reconstruction.

Titanium mesh cages (TMCs) have been used widely for the reconstruction of anterior column. The primary function of TMCs is to provide structural support to 
the anterior spinal column, which is known to transmit $80 \%$ of the axial load applied to the spine. ${ }^{7}$ Also TMCs have the potential advantages to support the restoration of anterior column stability after corpectomy in the thoracolumbar spine. Multiple cages with varying diameters and heights are available and can be filled with all autogenous graft (if available) which also enable to maintain osteoinductivity and osteoconductivity. ${ }^{8}$

The purpose of this study is to evaluate the radiologic stability of TMCs when used for corpectomy reconstruction of thoracolumbar spine and to review the clinical and neurological outcomes.

\section{Material and Methods}

\section{Patients}

A series of patients who underwent reconstruction of the anterior spinal column after thoracolumbar vertebrectomy between July 2000 and February 2005 were reviewed. Sixteen patients underwent surgery using a TMC for anterior spinal reconstruction, and the data from the patients' medical records and radiographs including immediate postoperative and follow-up radiographs were retrospectively analyzed. The primary diagnoses were acute fracture $(n=13)$, post-traumatic deformity $(n=3)$. There are 9 men and 7 women and the age ranged between 21 and 79 years (mean 40 years). An average duration of follow-up was 28.2 months (13 57 months). Single level reconstruction $(\mathrm{T} 12=3, \mathrm{~L} 1=4$, $\mathrm{L} 1=3, \mathrm{~L} 3=3, \mathrm{~L} 4=2$ ) was performed in 15 patients and two-level reconstruction (L1 \& L3) in one patient.

\section{Methods}

\section{1) Surgical technique}

All patients underwent corpectomy and decompression of the spinal canal with anterior column reconstruction using a cylindrical titanium mesh cage (Harms cage, Depuy-Acromed, Raynhem, MA). The surgical approach was anterior, via transthoracic, thoracoabdominal (retroperitoneal) or transperitoneal exposure ap- propriate to the level of corpectomy. The left side was the favored approach in thoracic and the right side in thracoabdominal (retroperitoneal) approach. Great care was taken to preserve the bony endplate as much as possible while preparing the endplates. After corpectomy, the inferior and superior ends of the TMCs were trimmed to match the sagittal alignment of the vertebral endplates. Each cage was loosely filled and then surrounded laterally and anteriorly with autologous bone chips (iliac graft $=2, \mathrm{rib}=1$ and the others used the fracture site autologous bone with demineralized bone matrix (Grafton $\left.{ }^{(}\right)$) added). Autograft was harvested from the fractured vertebral body in patients with burst fracture. Rib graft was used to supplement the vertebral body when a thoracoabdominal approach was used. If necessary, iliac bone graft was used to fill the TMCs. In acute burst fractures, the subtotal corpectomy was performed, leaving a small rim of vertebral cortex on the contralateral side to the approach, so as to avoid injury to the contralateral segmental vessels.

Additional stabilization devices were used in 14 patients: anterior double screw and rods system $(n=2)$, a single screw and rod system $(n=11)$, and anterior plate alone $(n=1)$. In two cases of acute fractures, no anterior stabilization device was used initially. However, subsequent posterior stabilization was necessary in two patients of acute burst fracture because of delayed kyphotic angulation.

\section{2) Radiologic evaluation}

Kyphotic deformity was assessed on lateral radiographs of thoracolumbar spine using the Cobb method. Cobb angle was measured between the superior endplate of upper level vertebra to the corpectomy and the inferior endplate of lower level vertebra to the corpectomy (Fig. 1). ${ }^{9}$ Kyphotic angle was measured preoperative, early postoperative and at the latest follow-up. The average correction in degrees and the loss of correction were calculated accordingly.

We checked the construct height of involved vertebrae by measuring the distance between the inferior 
endplate of superior vertebra to corpectomy and the inferior endplate of inferior vertebra to the corpectomy on the lateral X-ray. Subsidence of each TMCs was also checked by measuring the distance from the anterior aspect of the upper end plate of the superior vertebral body to the inferior endplate of the caudal vertebral body involved in the anterior fusion. Subsidence over the time was measured by the difference between the cage subsidence on initial postoperative X-rays and final follow-up films (Fig. 2).

The evaluation of osseous fusion on the final radiographs was assessed, according to the grading system advocated by Bridwell et al. ${ }^{2}$ Grade I indicates definite fusion (fused with remodeling and trabeculae present); Grade II indicates probable fusion (graft intact, not

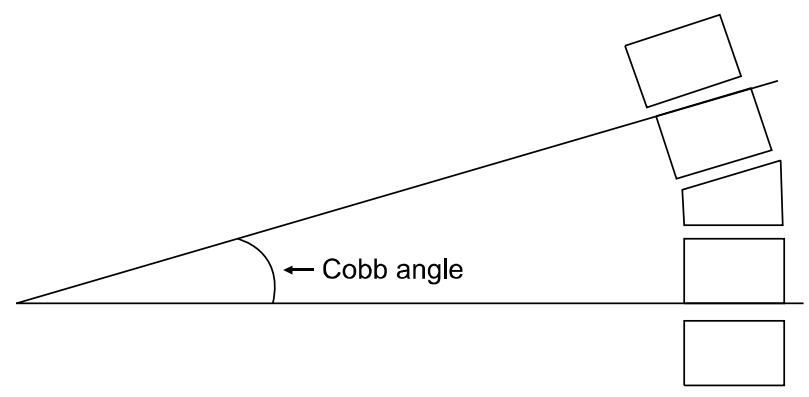

Fig. 1. Illustration for Cobb angle measurement. fully remodeled, no areas of lucency); Grade III indicates unlikely fusion (graft intact but lucency where it contacted the host bone surface); Grade IV indicates non-union (graft bone resorbed); and Grade V indicates that fusion could not be assessed. These measurements were performed by a single independent observer who was not involved in the surgery or care of these patients.

\section{3) Clinical assessment}

Neurologic status was assessed preoperatively, postoperatively, and at the latest follow-up according to the American Spinal Cord Injury Association modified Frankel Impairment Scale. Grade A represents a complete spinal cord injury in individuals in whom S4-5 sensory or motor function is absent, Grade B is an incomplete injury in whicht sensory but not motor function is preserved below the neurologic level and includes the sacral segments S4-5. Grade C is an incomplete injury in which motor function is preserved below the neurologic level and more than half of key muscles below the neurologic level have a muscle grade less than 3. Grade D is incomplete injury in which motor function is preserved below the neurologic level and at least half of key muscles below the neurologic level
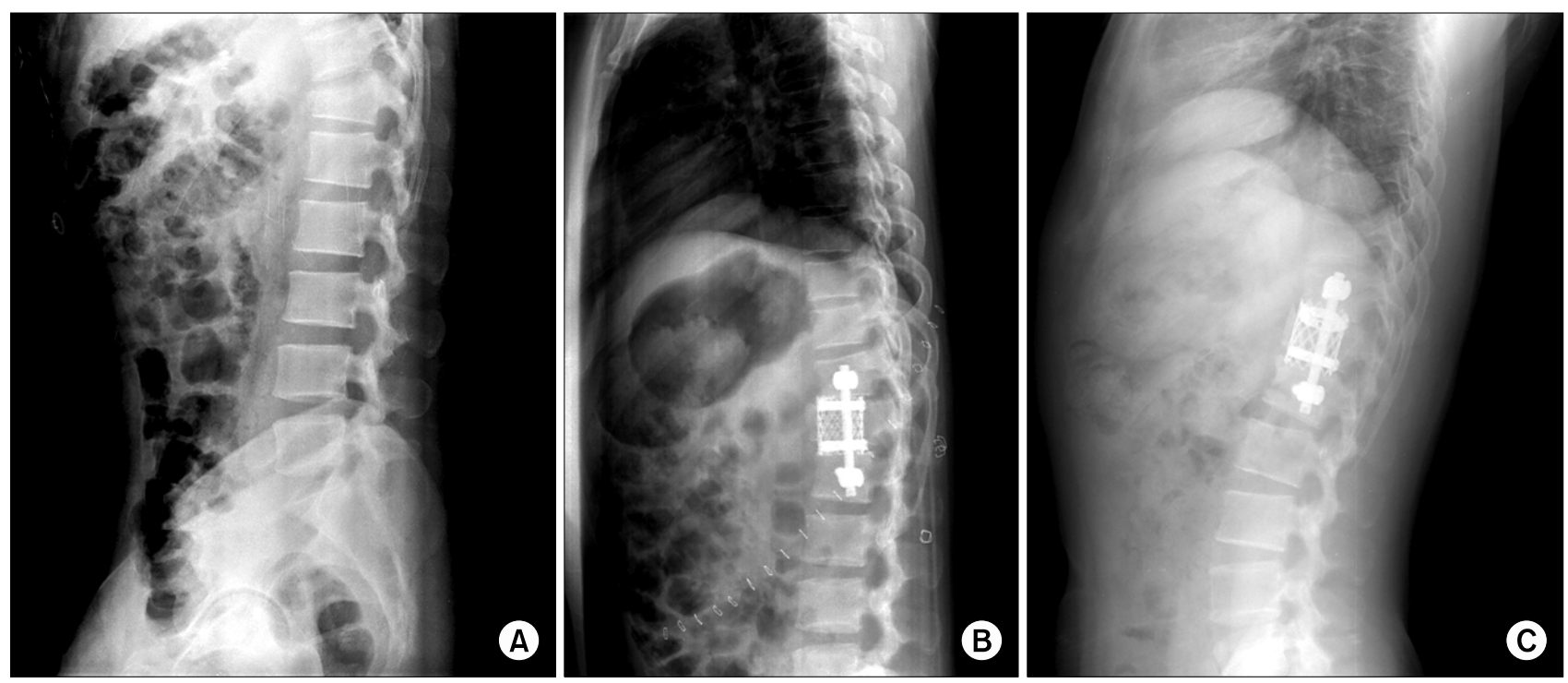

Fig. 2. Preoperative (A), early postoperative (B) and 14 months (C) follow-up lateral radiographs of a 29-years-old man with T12 burst fracture treated with titanium mesh cage with single screw and rod system after corpectomy. 
have a muscle grade greater than or equal to 3. And Grade E represents normal motor function and sensation.

\section{4) Statistical analysis}

Descriptive statistics (means and standard deviations) were obtained for quantitative variables and percentages were calculated for categorical variables of interest. Group comparisons of quantitative data were carried out using Wilcoxon signed rank test for independent samples. A $\mathrm{p}$ value of $<0.05$ was considered significant.

\section{Results}

\section{Radiologic results}

The mean ( \pm standard deviation) preoperative kyphosis was $2.0 \pm 15.2^{\circ}$. The mean kyphosis immediately after surgery was $-2.6 \pm 16.7^{\circ}$. The mean kyphosis at final follow-up was $3.4 \pm 15.3^{\circ}$. The mean loss of correction at final follow-up was $6.0 \pm 1.4^{\circ}(\mathrm{p}=0.003$, preoperative versus postoperative; $p=0.935$, preoperative versus final follow-up). There was no significant difference between the postoperative and final kyphosis measurements in this study. There was a significant difference between the preoperative versus postoperative kyphotic angle in acute fracture $(\mathrm{p}=0.004)$; however, no statistical significance in post-traumatic deformity $(p=0.109)$ (Table 1). The mean preoperative height was $41.6 \pm$ $14.5 \mathrm{~mm}$. The mean height immediately after surgery

Table 1. Kyphotic angle measurements before, after and at final follow-up

\begin{tabular}{|c|c|c|c|c|}
\hline & $\begin{array}{c}\text { Preoperative } \\
\text { kyphosis }\left({ }^{\circ}\right)\end{array}$ & $\begin{array}{c}\text { Postoperative } \\
\text { kyphosis }\left({ }^{\circ}\right)\end{array}$ & $\begin{array}{l}\text { Follow-up } \\
\text { kyphosis( }\left(^{\circ}\right)\end{array}$ & $p$ value \\
\hline $\begin{array}{l}\text { All patients } \\
(n=16)\end{array}$ & $\begin{array}{c}3.9 \\
(-29.0 \sim 29.5)\end{array}$ & $\begin{array}{c}-2.6 \\
(-34.0 \sim 25.6)\end{array}$ & $\begin{array}{c}3.4 \\
(-24.0 \sim 28.5)\end{array}$ & $\begin{array}{l}0.003^{*} \\
0.935^{\dagger}\end{array}$ \\
\hline $\begin{array}{l}\text { Acute fracture } \\
(n=13)\end{array}$ & 1.2 & -7.8 & -0.9 & $0.004^{*}$ \\
\hline $\begin{array}{l}\text { Post-traumatic } \\
\text { deformity }(n=3)\end{array}$ & 5.0 & -2.2 & 1.3 & $0.109^{*}$ \\
\hline
\end{tabular}

Values are means (range). ${ }^{*}$ Comparison of preoperative and postoperative measurement; ${ }^{\dagger}$ Comparison of preoperative and follow-up measurement. was $47.9 \pm 14.8 \mathrm{~mm}$ and at final follow-up was $41.5 \pm$ $15.7 \mathrm{~mm}(\mathrm{p}<0.0001$, preoperative versus postoperative; $\mathrm{p}=0.958$, preoperative versus final follow-up). The mean loss of correction at final follow-up was $6.4 \pm 0.8$ $\mathrm{mm}$ (Table 2). At final follow-up, cage subsidence was measured to be an average of $5.7 \pm 2.8 \mathrm{~mm}$. The fusion status on the final radiographs revealed Grade I fusion in 6, Grade II fusion in 9 and Grade V fusion (unable to be assessed) in 1 patient.

\section{Clinical results}

The preoperative neurologic status was normal in 4 , paraparesis in 11, and 1 patient was completely paraplegic. At the final follow up, Eleven of 16 patients (69\%) with incomplete injuries improved a minimum of one grade on the ASIA modified Frankel Scale. One patient categorized as modified Frankel A demonstrated some improvement, whereas all four neurologically intact patients remained unchanged. There was no case of neurological deterioration following anterior decompression and stabilization (Table 3).

Table 2. Height measurements before, after and at final follow up

\begin{tabular}{lcccc}
\hline & $\begin{array}{c}\text { Preoperative Postoperative } \\
\text { construct } \\
\text { height }(\mathrm{mm})\end{array}$ & $\begin{array}{c}\text { Follow-up } \\
\text { construct } \\
\text { height }(\mathrm{mm})\end{array}$ & $\begin{array}{c}\text { construct } \\
\text { height }(\mathrm{mm})\end{array}$ & $\mathrm{p}$ value \\
\hline $\begin{array}{c}\text { All patients } \\
(\mathrm{n}=16)\end{array}$ & 41.6 & 47.9 & 41.5 & $<0.0001^{*}$ \\
$\begin{array}{c}\text { Acute fracture } \\
(\mathrm{n}=13)\end{array}$ & $47.0 \sim 60.0)$ & $\left(\begin{array}{c}19.3 \sim 68.0) \\
(16.2 \sim 61.3)\end{array}\right.$ & $0.958^{+}$ \\
$\begin{array}{c}\text { Post-traumatic } \\
\text { deformity }(\mathrm{n}=3)\end{array}$ & 32.4 & 42.4 & 48.5 & $0.004^{*}$ \\
\hline
\end{tabular}

Values are means (range). "Comparison of preoperative and postoperative measurement; ${ }^{\dagger}$ Comparison of preoperative and follow-up measurement.

Table 3. ASIA Modified Frankel Grades at admission and at discharge

\begin{tabular}{ccccccc}
\hline & & \multicolumn{5}{c}{ ASIA grade at discharge } \\
\cline { 3 - 7 } ASIA & No. of patients & No of patients \\
grade & at admission & A & B & C & D & E \\
\hline A & 1 & & 1 & & & \\
B & 2 & & & & 2 & \\
C & 6 & & 3 & 3 & \\
D & 3 & & & 2 & 1 \\
E & 4 & & & & & 4 \\
\hline
\end{tabular}


Table 4. Perioperative complications

\begin{tabular}{lc}
\hline \multicolumn{1}{c}{ Complications } & No. of patients \\
\hline Postoperative pneumonia & 1 \\
Neurogenic bladder & 1 \\
Sepsis (neglected preoperatively) & 1 \\
Superficial wound infection & 1 \\
Cage malposition & 1 \\
Screw malposition & 1 \\
Pancytopenia & 1 \\
\hline
\end{tabular}

\section{Perioperative complications}

Perioperative complications included postoperative pneumonia $(\mathrm{n}=1)$, neurogenic bladder $(\mathrm{n}=1)$, undiscovered preoperative sepsis $(n=1)$, superficial wound infection $(n=1)$, cage or screw malposition $(n=2)$ and pancytopenia due to tegretol $(n=1)$ (Table 4). One patient with acute burst fracture was in poor general condition with unfavorable laboratory findings, which was neglected preoperatively. The general condition aggravated despite of antibiotics treatment and expired 1 month after surgery. All complications were related to treatment of the underlying disease, and in none of the cases was found complications directly attributable to the use of TMCs. There were 2 cases of cage and screw malposition, and revision procedure was performed two days later. There was no case of cage fracture.

\section{Discussion}

Pathologic processes such as trauma, malignancy, infection, and congenital/developmental abnormalities can involve the vertebral bodies, resulting in incapability of maintaining anterior column support and stability. ${ }^{3,4,10,11}$ Anterior approaches can achieve sufficient decompression and immediate stabilization of the spinal canal. ${ }^{6}$ The ideal reconstruction device of anterior column should provide a mechanical stability and spinal alignment maintenance while facilitating stable fusion. Structural autografts have been the gold standard in anterior reconstruction. Tricortical iliac grafts, fibula and rib have been used extensively. ${ }^{12-14}$ Disadvantages of them are less ideal shape for corpectomy reconstruction, lack of intrinsic stability and high rates of bone graft donor site morbidity. ${ }^{5,15}$ Other available options include structural allograft or prosthetic implants such as TMCs. Allograft bone allows more suitable bone shape with cylindrical mesh structure. Although the allograft bone may provide more flexibility in dictating the graft length and configuration, graft collapse, graft fracture, nonunion, the limited availability, high cost, and a risk of disease transmission may impose some constraints on its use. ${ }^{16}$

TMCs with cancellous autograft bone after corpectomy of the thoracolumbar spine provide immediate structural support to the anterior column. TMCs provide structural support while the cancellous bone inside the cage promotes fusion. Hollow cylindrical mesh structure can easily adjust the size, and reuse of autogenous cancellous bone in acute thoracolumbar fracture without the morbidity associated with harvesting of autograft. ${ }^{8}$ The cages give resistance to axial compression, lateral flexion and axial rotation, and also have resistance to toggling (translation of vertebra above \& below the cage). ${ }^{15}$ The additional stability can be achieved with anterior or posterior stabilization device. The distraction forces are resisted by the soft tissue and additional stabilization devices.

This study demonstrates that TMCs was effective at maintaining sagittal alignment over a postoperative period of 28 months. The clinical outcomes were compatible with the published reports using TMCs. ${ }^{1,8,12}$ The only significant difference between the preoperative versus postoperative kyphotic angle was detected and other parameters showed no significance. In acute trauma, correction of kyphotic angle and height were significant at early postoperative period. However, the corrected angle and height had been lost slowly as time goes by within acceptable range. In contrast to acute trauma, kyphotic angle and height in post-traumatic deformity were not corrected in immediately postoperatively and 
at last follow-up. This finding might be due to more elderly patients in both groups, and osteoporosis were thought to be an obstacles for the stable reconstruction. Despite of shortcomings in this study, the clinical and radiologic success of TMCs in acute fracture supports that ongoing use of the TMC after thoracolumbar corpectomy is recommended. In post-traumatic deformity, the efficacy of TMCs was indeterminable due to a relative small number of patient group and short follow-up periods, and it has to be elucidated in the future.

The subsidence of the Titanium mesh cage into the adjacent endplates may represent increased load at the cage-endplate interface or reduced bone density. The sharp edges in the cut ends of the cage may contribute to some early subsidence as the sharp edge breach the surface of the endplate. Significant osteoporosis might be a contraindication to the use of mesh cages. ${ }^{17}$ Most of patients were not evaluated for bone density before surgery in our study. Mean subsidence in this study was $5.7 \mathrm{~mm}(1.6 \sim 10.9 \mathrm{~mm})$, which was somewhat larger than previous studies. However, we did not observe the severe collapse or significant recurrence of deformity.

In our investigation, fusion status was assessed using plain radiographs, according to the grading system advocated by Bridwell et al. ${ }^{2}$ We have not closely evaluated radiologic fusion but rather accepted radiologic stability when the clinical results have been acceptable. Because it is well established that radiologic methods of fusion assessment are not completely reliable and the only accurate method for assessing fusion is surgical exploration. One study by Blumental and Gill compared radiological fusion assessment with surgical exploration and revealed $69 \%$ correlation between them. ${ }^{1}$ The fusion mass outside and surrounding the cages is easier to assess with plain radiography than the fusion within the cages. Thus, we filled the bone chips around the cages to help the assessment later. Bone fusion within the cage may be assessed with CT. The 1-mm slice CT scan with coronal and sagittal reconstructions is superior to plain radiographs for assessment of fusion within and around the cages. We did not check follow-up CT scan routinely. It has become our routine to check follow-up CT scan for fusion including settling/loss of correction on sagittal and axial plane.

In two acute fractures, no anterior stabilization device was used initially. Two cases in acute burst fracture in L4 developed a progressive kyphosis within a month. Because of anterior tilting of the cage and instability, additional posterior screw fixation was necessary. The authors always augment vertebral reconstruction with additional stabilization in the acute burst fracture in an attempt to provide the mechanically stable construct. Anterior, posterior or combined anterior and posterior procedures have been advocated by different authors and show various degrees of success. ${ }^{7,16}$ Dvorak et al. recommended anterior and posterior instrumentation in thoracic vertebral reconstruction to avoid the mechanical failure. ${ }^{7}$ As a result of biomechanical improvements in anterior instrumentation, several investigators reported good results with anterior decompression and standalone instrumentation. ${ }^{5,6,18}$ Brodke et al. reported that both the plate and rod-style systems are able to stabilize a corpectomy reconstruction model for the four axes of axial compression, flexion-extension, lateral bending, and axial rotation. ${ }^{19}$ But TMCs without additional supplementary instrumentation do not adequately support the anterior column and should be avoided especially in the acute burst fracture. The goals of treatment in patients with unstable thoracolumbar acute burst fracture and post-traumatic deformity should be achievement of spinal alignment and decompression of neural elements, stabilization of an unstable fracture and prevention of further progressive deformity or neurological injury as well as the provision of a painless, functional spine, and maximization of neurological and clinical recovery. In this point of view, some kind of kyphosis and subsidence at the last follow-up were observed but there was no clinical and neurological aggravation in all cases with anterior instrumentation in this study. Therefore, we suggest that anterior instrumentation without combined instrumentation can provide a good chance of stabilization for anterior spinal column. 
In conclusion, although there were $6^{\circ}$ of kyphosis recurrence and a 5.7 millimeters of cage settling in many cases at final follow-up radiographs, significant recurrence of deformity did not occur. There was no case of cage migration or failure. TMCs offer excellent biomechanical stability without evidence or any significant recurrence of deformity. In the case of acute fracture and post-traumatic deformity, TMCs with anterior instrumentation allows a good structural support and maintain spinal alignment.

\section{References}

1. Blumenthal SL, Gill K. Can lumbar spine radiographs accurately determine fusion in postoperative patients? Correlation of routine radiographs with a second surgical look at lumbar fusions. Spine 1993; 18:1186-9.

2. Bridwell KH, Lenke LG, McEnery KW, Baldus C, Blanke K. Anterior fresh frozen structural allografts in the thoracic and lumbar spine. Do they work if combined with posterior fusion and instrumentation in adult patients with kyphosis or anterior column defects? Spine 1995;20:1410-8.

3. Finkelstein JA, Chapman JR, Mirza S. Anterior cortical allograft in thoracolumbar fractures. J Spinal Disord 1999;12:424-9.

4. Hussein AA, El-Karef E, Hafez M. Reconstructive surgery in spinal tumors. Eur J Surg Oncol 2001;27:196-9.

5. Sasso RC, Best NM, Reilly TM, McGuire RA Jr. Anterior-only stabilization of three- column thoracolumbar injuries. J Spinal Disord Tech 2005;18(Suppl):S7-14.

6. Kostuik JP. Anterior fixation for fractures of the thoracic and lumbar spine with or without neurologic involvement. Clin Orthop Relat Res 1984;189:103-15.
7. Dvorak MF, Kwon BK, Fisher CG, Eiserloh HL 3rd, Boyd M, Wing PC. Effectiveness of titanium mesh cylindrical cages in anterior column reconstruction after thoracic and lumbar vertebral body resection. Spine 2003;28:902-8.

8. Robertson PA, Rawlinson HJ, Hadlow AT. Radiologic stability of titanium mesh cages for anterior spinal reconstruction following thoracolumbar corpectomy. J Spinal Disord Tech 2004;17:44-52.

9. Hong JT, Lee SW, Son BC, Sung JH, Park CK, Kim MC. Kyphotic angle measurement accuracy for vertebral osteoporotic compression fracture: reliable method for kyphotic angle measurement. $J$ Korean Neurosurg Soc 2006;39:256-9.

10. Roberson JR, Whitesides TE Jr. Surgical reconstruction of late posttraumatic thoracolumbar kyphosis. Spine 1985;10:307-12.

11. Streitz W, Brown JC, Bonnett CA. Anterior fibular strut grafting in the treatment of kyphosis. Clin Orthop Relat Res 1977;128:140-8.

12. Eck KR, Bridwell KH, Ungacta FF, Lapp MA, Lenke LG, Riew KD. Analysis of titanium mesh cages in adults with minimum two-year follow-up. Spine 2000;25:2407-15.

13. Molinari RW, Bridwell KH, Klepps SJ, Baldus C. Minimum 5-year follow-up of anterior column structural allografts in the thoracic and lumbar spine. Spine 1999;24:967-72.

14. Singh K, DeWald CJ, Hammerberg KW, DeWald RL. Long structural allografts in the treatment of anterior spinal column defects. Clin Orthop Relat Res 2002;394:121-9.

15. Lee SW, Lim TH, You JW, An HS. Biomechanical effect of anterior grafting devices on the rotational stability of spinal constructs. $J$ Spinal Disord 2000;13:150-5.

16. Vahldiek MJ, Panjabi MM. Stability potential of spinal instrumentations in tumor vertebral body replacement surgery. Spine 1998;23:543-50.

17. Hasegawa K, Abe M, Washio T, Hara T. An experimental study on the interface strength between titanium mesh cage and vertebra in reference to vertebral bone mineral density. Spine 2001;26:957-63.

18. Schnee CL, Ansell LV. Selection criteria and outcome of operative approaches for thoracolumbar burst fractures with and without neurological deficit. J Neurosurg 1997;86:48-55.

19. Brodke DS, Gollogly S, Bachus KN, Alexander Mohr R, Nguyen BK. Anterior thoracolumbar instrumentation: stiffness and load sharing characteristics of plate and rod systems. Spine 2003;28:1794-801. 